\title{
Screening for bacteriuria in patients with spinal cord lesion: Dipstick test, microscopic examination and urine culture
}

\author{
$\mathrm{KL}_{\text {Faarvang }}{ }^{1}$, P Müller ${ }^{1}$, B Lomberg ${ }^{1}$ and F Biering-Sørensen ${ }^{*}, 1$ \\ ${ }^{1}$ Centre for Spinal Cord Injured, The Neuroscience Centre, Rigshospitalet, Copenhagen University Hospital, Denmark
}

\begin{abstract}
Aim: To investigate the positive and negative predictive values for bacteriuria of a rapid chemical dipstick procedure for leukocytes and nitrite, and a microscopic examination for leukocytes and bacteria with a urine culture being the reference in patients with spinal cord lesion (SCL).

Methods: A prospective study. Significant bacteriuria was defined as $\geqslant 10^{5} \mathrm{CFU} / \mathrm{mL}$. The microscopic examination for leukocytes was divided in four different cut-off limits for positive results.

Material: A total of 256 consecutive early morning urine samples were collected from 143 SCL patients admitted to our in-patient facility.

Results: One hundred and twenty-eight urine cultures revealed significant bacteriuria. Eightyseven were infected by only one microorganism, 41 samples contained significant growth of more than one species. A total of 186 microorganisms in significant growth were identified. Conclusion: Three or more leukocytes should be considered as a positive result. The dipstick and microscopy tests are equally valuable, considering the single tests as well as the combinations evaluated.

Spinal Cord (2000) 38, $106-108$
\end{abstract}

Keywords: spinal cord injury; bacteriuria; dipstick test; urine microscopy; urine culture

\section{Introduction}

Urinary tract infections are important contributors to morbidity in patients with spinal cord lesion (SCL). Furthermore, as recurrent infections of the lower urinary tract are believed to predispose to the renal insufficiency that continues to be one of the serious complications of SCL, careful screening for bacteriuria should be performed regularly.

This study was undertaken to examine and compare two screening methods in daily routine practice, microscopic urine analysis and a dipstick procedure, with a well recognised reference method, bacterial culture. The reason for this was to clarify if either of the screening methods was superior or if they could replace each other in this particular patient population.

As it is known the efficacy of dipsticks will vary with the prevalence of bacteriuria in different patient populations. ${ }^{1,2}$ It is of importance to evaluate such tests in the population concerned, in this case SCL patients.

*Correspondence: F Biering-Sørensen, Centre for Spinal Cord Injured, The Neuroscience Centre, Rigshospitalet, Copenhagen University Hospital, Havnevej 25, DK-3100 Hornbæk, Denmark

\section{Patients and methods}

Patients

Urine samples were obtained from 143 SCL patients admitted to our hospital's in-patient facility.

\section{Urine samples}

A total of 256 consecutive early morning urine samples were collected by a standardised 'clean' technique. If the patient used a catheter intermittently the urine was taken directly from the catheter during emptying of the bladder. If the patient did not empty with a catheter an autoclaved bedpan or bed bottle was used to collect the urine. The sampling technique with a single sample for screening resembles the daily routine in the centre. Part of the urine sample was immediately placed at $4{ }^{\circ} \mathrm{C}$ and analyses with chemical dipstick and microscopy were performed within $3 \mathrm{~h}$. The sample was thoroughly turned before analysis. The remainder of the sample was used for urine culture.

Urine culture

Quantitative bacterial cultures were grown on standard media. Significant bacteriuria was in the present study defined as $\geqslant 10^{5}$ colony forming units per millilitre. 


\section{Screening Tests}

Dipstick chemical urine analysis The Nephurtest + leuco (Böhringer Mannheim, Germany) was used. It can detect leukocytes esterase, nitrite, $\mathrm{pH}$, glucose, protein and blood. Only leukocyte esterase and nitrite were evaluated in this study.

Leukocytes The reaction reveals the presence of esterase that occurs in neutrophil granulocytes. These esterases cleave in indoxyl ester, and the indoxyl so liberated reacts with a diazonium salt to produce a violet dye. Colourations can be judged after 1-2 min. Colour blocks show a positive or negative result. Therapy with nitrofurantoin and a large intake of vitamin $\mathrm{C}$ can affect the result.

Nitrite The reaction reveals the presence of nitrite and then indirectly of nitrite-forming bacteria (mainly those of the Enterobacteriaceae) in the urine. The test shows ,+++ or +++ ; all three were considered positive. Storage of urine in the bladder for $4-8 \mathrm{~h}$ is essential for the production of detectable nitrite concentrations.

Urine microscopy Routine microscopy of the unstained sediment in a highpower field (400 times) after centrifugation for 4-5 min at 1500 rounds per min was performed. The leukocytes per visual field were counted, four different cut-off limits for positive results were tested. Regarding bacteria, if any was observed, the test was considered positive.

\section{Statistical methods}

The results of urine culture were used as reference for the screening tests, chemical dipstick and microscopy. To evaluate the strength of the screening tests the predictive values were calculated as follows: The predictive value of a positive result (PV pos), ie, the number of the true positive results of the test divided by all the positive results of the test (positive predictive value $=$ true positive $/($ true positive + false positive) $)$. The predictive value of a negative result (PV neg), ie the number of true negative results divided by all negative results (negative predictive value $=$ true negative/(true negative + false negative)). In addition the $95 \%$ confidence limits are given.

\section{Results}

Out of 256 urine samples examined by routine clinical laboratory culture, revealed 128 significant bacteriuria. Of the 128 positive samples, 87 contained only one microorganism, whereas 41 samples contained significant growth of more than one species. Thus a total of 186 microorganisms in significant growth were identified. The most common species found were $E$. coli, streptococcus species, enterococcus species and pseudomonas species. The detailed results are shown in Figure 1.
The microscopic examination for leukocytes were divided in four different cut-off limits for positive results in order to test which level was most suited for our patients. Based on the PV pos and PV neg values we concluded from the results given in Figure 2 that three or more leukocytes should be considered as a positive result. This is also the level recommended by our clinical biochemical laboratory. Therefore this cutoff limit is used in the study.

With the results of urine culture as the reference, the predictive values of the tests-chemical dipstick and microscopy-were calculated. The results, including combinations of different test procedures, are shown in Figure 3. From these results it can be concluded, that the dipstick and microscopy tests are equally valuable, considering both the single and combined tests.

\section{Indentified microorganisms}
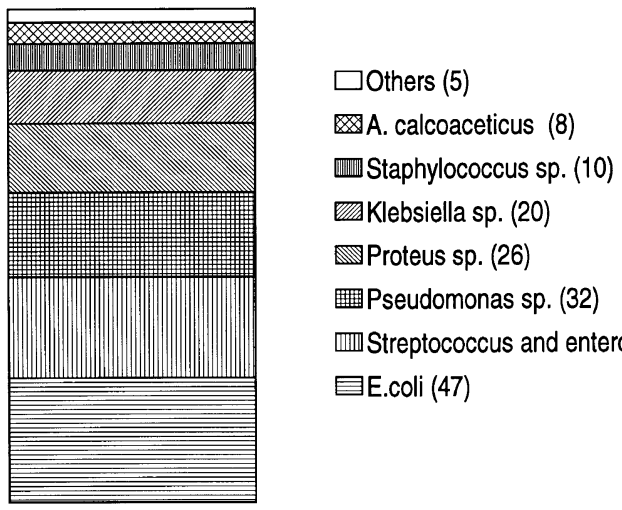

血Streptococcus and enterococcus sp. (38)

目E.coli (47)

Figure 1 Microorganisms identified in 128 urine samples with $\geqslant 100000$ colony forming units per millilitre. In 87 samples only one microorganism was found, whereas 41 samples contained significant growth of more than one species. A total of 186 microorganisms were isolated

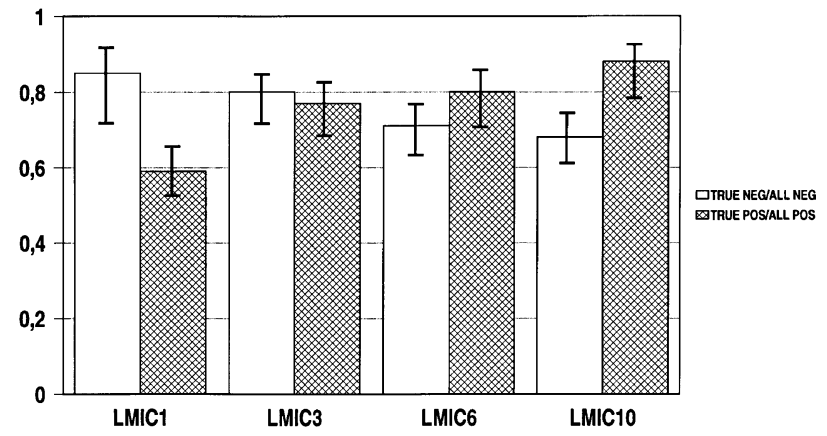

Figure 2 Microscopic examination for leukocytes: Four different cut-off limits for positive results have been tested in order to see which level was most suited for our patients. The 95\% confidence limits are given: LMIC1: One or more leukocytes per field of vision. LMIC3: Three or more leukocytes per field of vision. LMIC6: Six or more leukocytes per division. LMIC10: Ten or more leukocytes per field of vision 


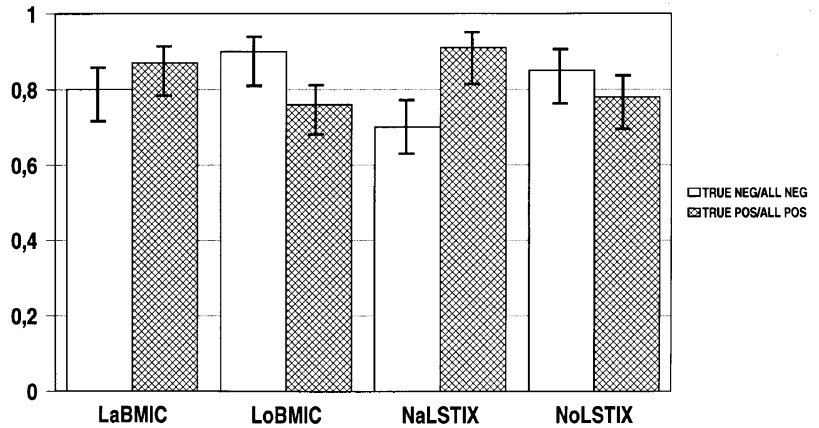

Figure 3 Positive and negative predictive values of test procedures, either single or in combination. The $95 \%$ confidence limits are given. LaBMIC: both $3 \geqslant$ leukocytes per field of vision and microscopic detection of bacteria positive to be considered a positive result. LoBMIC: $3 \geqslant$ leukocytes per field of vision and/or microscopic detection of bacteria positive to be considered a positive result. NaLSTIX: both dipstick test for nitrate and dipstick test for leukocyte esterase positive to be considered a positive result. NoSTIX: dipstick test for nitrite and/or dipstick test for leukocyte esterase positive to be considered a positive result

\section{Discussion}

The bacteria isolated from the urine in the present study (Figure 1) showed a pattern characteristic for a SCL patient population. ${ }^{3}$

The efficacy of microscopy as a screening test for bacteriuria varies widely: sensitivities range from $60 \%$ to $100 \%$ and specificities from $49 \%$ to $100 \%{ }^{4}$. A major factor is the lack of standardisation both in technique and in the criteria for defining abnormal results; however, if these are carefully standardised, a sensitivity and specificity up to $95 \%$ can be attained. ${ }^{4}$ Falsely negative urine leukocytes counts are caused by loss of leukocytes during the centrifugation of the urine, and a delayed examination of urine can result in a reduction in sensitivity due to lysis of leukocytes. ${ }^{5}$ Microscopy as a screening test for bacteriuria and leukocyturia requires special equipment and training of personnel.

The dipstick test for leukocytes can detect lysed leukocytes. A negative nitrite test despite significant bacteriuria may be explained by the presence of bacteria that do not form nitrite. Nondetectable nitrite levels may also be caused by high diureses, dilution, starvation or frequent voiding. Storage of urine in the bladder for $4-8 \mathrm{~h}$ is essential for the production of detectable nitrite concentrations. Therefore it is essential to collect a morning urine.

The efficacy of dipsticks will vary with the different patient populations ${ }^{1,2}$ because the predictive value is highly dependent on the prevalence of 'disease' in the patient population studies, therefore such tests must be evaluated in specific groups as in our study. With the same definition of bacteriuria comparable results have previously been found for the use of dipsticks in SCL individuals. ${ }^{6}$

We conclude that the screening procedures, microscopic examination and chemical dipstick test were equally valuable in predicting significant bacteriuria, considering the single test as well as the combinations given. In industrialised countries where manpower is expensive the dipstick tests can safely and efficiently replace the relative time-consuming microscopic procedure. On the other hand in countries with low salaries the expense of dipstick tests can seem very high, and the microscopy screening test will be just as good when performed by trained personnel. For both tests the necessary precautions mentioned have to be taken to obtain reliable results.

\section{References}

1 Bank CM, Codrington JF, van Diejen-Visser MP, Brombacher JP. Screening urine specimen populations for normality using different dipsticks: evaluation of parameters influencing sensitivity and specificity. J Clin Chem Biochem 1987; 25: 299-307.

2 Kromann-Andersen B, Walter S, Jakobsen H, Vejlsgaard R. Detection of neutrophilocytes and $\mathrm{NO}_{2}$-producing bacteria in urine by dipstick BM-test-9. Dan Med Bull 1987; 34: 182-183.

3 Biering-Sørensen $\mathrm{F}$ et al. Ciprofloxacin as prophylaxis for urinary tract infection; prospective, randomized, cross-over, placebo controlled study in patients with spinal cord lesion. $J$ Urol 1994; 151: 105 - 108. [Erratum J Urol 1994; 151: 1032].

4 Jenkins RD, Fenn JP, Matsen JM. Review of urine microscopy for bacteriuria. JAMA 1986; 255: 3397-3403.

5 Kierkegaard $\mathrm{H}$ et al. Falsely negative urinary leucocyte counts due to delayed examination. Scand J Clin Lab Invest 1980; 40: $259-261$.

6 Tuel SM, Meythaler JM, Cross LL, McLaughlin S. Cost-effective screening by nursing staff for urinary tract infection in the spinal cord injured patient. Am J Phys Med Rehabil 1990; 69: 128-131. 